\title{
Chromatic aberration effect in refraction of spin waves
}

\author{
Tian Li*, Takuya Taniguchi**, Yoichi Shiota*, Takahiro Moriyama*, and Teruo Ono*,*** \\ *Institute for Chemical Research, Kyoto University, Uji, Kyoto 611-0011, Japan \\ ${ }^{* *}$ Fakultãt für Physik, Technische Universität München, Garching 85748, Germany \\ ${ }^{* * *}$ Center for Spintronics Research Network (CSRN), Graduate School of Engineering Science, Osaka University, Osaka 560-8531, \\ Japan
}

We numerically investigated the refraction property of spin waves (SWs) at thickness step in films with out-ofplane magnetization, in which the SWs propagate isotropically in the film plane. It was confirmed the isotropic SWs were refracted at a thickness step by following the Snell's law. We also found that the refraction angle of SWs of the dipole-exchange mode depends on the resonant frequency, indicating that the chromatic aberration effect should be taken into account in designing magnonic devices.

Key words: magnonics, spin waves

Magnonics is a rapidly developing research field, in which spin waves (SWs) are utilized to transfer and process information ${ }^{1-4)}$. In magnonics, controlling the propagation direction of SWs is one of the crucial issues ${ }^{5-}$ 9). It has been recently demonstrated that refraction is also useful for controlling the propagation direction of $\mathrm{SWs}^{12,13,16-18)}$. In optics, the dispersion relation of light is isotropic, and thus, the relation between the incident and refraction angles is determined by the ratio of the refractive indices. On the other hand, for spin waves in thin films with in-plane magnetization the dispersion relation is anisotropic, and thus, deviations from Snell's law in optics has been reported $\left.{ }^{13}, 16\right)$. This deviation from Snell's law in optics results in the complicated design for magnonic devices ${ }^{11}$. Thus, isotropic SWs, rather than anisotropic SWs, are expected to be more suitable for application. Here, we numerically investigate the refraction property of SWs in thin films with perpendicular magnetization, in which the isotropic SW propagation in the film plane is expected from the symmetry. It is found that the refraction of SWs follows the Snell's law in optics with the chromatic aberration effect.

The micromagnetic simulation is performed utilizing mumax $3^{19)}$. In simulation, we use the following material parameters reported for yttrium iron garnet (YIG); the saturation magnetization $M_{\mathrm{s}}=139 \mathrm{kA} / \mathrm{m}$, the exchange stiffness $A_{\mathrm{ex}}=4.15 \mathrm{pJ} / \mathrm{m}$, and the Gilbert damping constant $1 \times 10^{-4} 20,21$ ). In this study, two types of samples, labeled as sample A and B, are designed. Both samples are shaped as displayed in Fig. 1(a) and consist of thicker and thinner regions. The thicknesses of thicker and thinner areas are set to be $800 \mathrm{~nm}$ and $400 \mathrm{~nm}$ in sample A, and set to be $100 \mathrm{~nm}$ and $50 \mathrm{~nm}$ in sample $\mathrm{B}$, respectively. The cell size for the calculation is $200 \times$ $200 \times 200 \mathrm{~nm}^{3}$ in sample A and $50 \times 50 \times 50 \mathrm{~nm}^{3}$ in sample B. Note that the cell size is set to be much smaller than the wavelength of excited SWs. To avoid the SW reflections at the edges of calculation area, the damping constants in the areas of $6 \mu \mathrm{m}$ width from the left and right edges are set to increase gradually to 1 . An external magnetic field $\mu_{0} H_{\text {ext }}=200 \mathrm{mT}$ is applied to $+\mathrm{z}$ direction, which is enough to saturate the magnetization to $\mathrm{z}$ direction, and SWs are excited by locally applied rf field $\mu_{0} H_{\mathrm{rf}} \sin (2 \pi f t)$ along $\mathrm{x}$ direction at an antenna in the thicker area. In this work, we use $\mu_{0} H_{\mathrm{rf}}=1 \mathrm{mT}$ and the frequency $f$ of $\mathrm{rf}$ field is varied as $f=0.9,1.0$, and 1.1 $\mathrm{GHz}$. We analyze the results recorded at $100 \mathrm{~ns}$ after the SW excitation.

Fig. 1(b) shows a typical result, which displays the phase of magnetization precession at each position in $x y$ plane. A SW with wavenumber $k_{1}$ propagates from the antenna to the thickness step with the incident angle $\theta_{1}$ and it is refracted into the other SW with wavenumber $k_{2}$ at the thickness step with the refraction angle $\theta_{2}$. The estimation of $k_{1}, k_{2}$, and $\theta_{2}$ is accomplished as follows. Firstly, we define two areas (A1 and A2) and perform two-dimensional fast Fourier transform (2D-FFT) on the data in each area. Results of 2D-FFT are shown in Figs. 1(c) and 1(d). Secondly, we estimate $k_{1}$, which is the incident wavenumber in $+x$ direction, from the data on $k_{y}=0$ (Fig. 1(c)). $k_{1}$ is given as the second highest peak, as shown in Fig. 1(e). Thirdly, we linearly fit the 2D-FFT results of $\mathrm{A} 2$ and obtain $\theta_{2}$ from the slope (Fig. 1(d)). Finally, $k_{2}$ is estimated from the second highest peak on the fitted line, as shown in Fig. 1(f).

To confirm that SWs propagate isotropically in both samples, we plot $k_{1}$ and $k_{2}$ as a function of $\theta_{1}$, as shown in Fig. 2. Both $k_{1}$ and $k_{2}$ are almost constant with respect to $\theta_{1}$ even if the resonant frequency is varied from $0.9 \mathrm{GHz}$ to $1.1 \mathrm{GHz}$. If the $\mathrm{SW}$ dispersion relation is anistropic in the film plane, the wavenumber should depend on the SW propagating dicrections ${ }^{13)}$. Therefore, the results shown in Fig. 2 indicates that the SWs propagate isotropically in both thicker and thinner regions, and Snell's law in optics, $\sin \theta_{1} / \sin \theta_{2}=k_{2} / k_{1}$, can be applied in the present system.

It is known that the dispersion relation of SW 
propagating in a film with perpendicular magnetization can be described as

$$
\omega^{2}=\left(\omega_{H}+\frac{2 A_{\mathrm{ex}} \gamma}{M_{s}} k^{2}\right)\left[\omega_{H}+\frac{2 A_{\mathrm{ex}} \gamma}{M_{s}} k^{2}+\omega_{M}\left(1-\frac{1-e^{-k d}}{k d}\right)\right] \text { (1) }
$$

where $\omega$ is the angular frequency of the excited SW, $\omega_{H}=\mu_{0} \gamma\left(H_{\text {ext }}-M_{\mathrm{s}}\right), \quad \omega_{M}=\mu_{0} \gamma M_{\mathrm{s}}, \quad \mu_{0} \quad$ is the permeability of vacuum, $\gamma$ is the gyromagnetic ratio, and $d$ is the film thickness ${ }^{10,22)}$. Here, both dipole-dipole
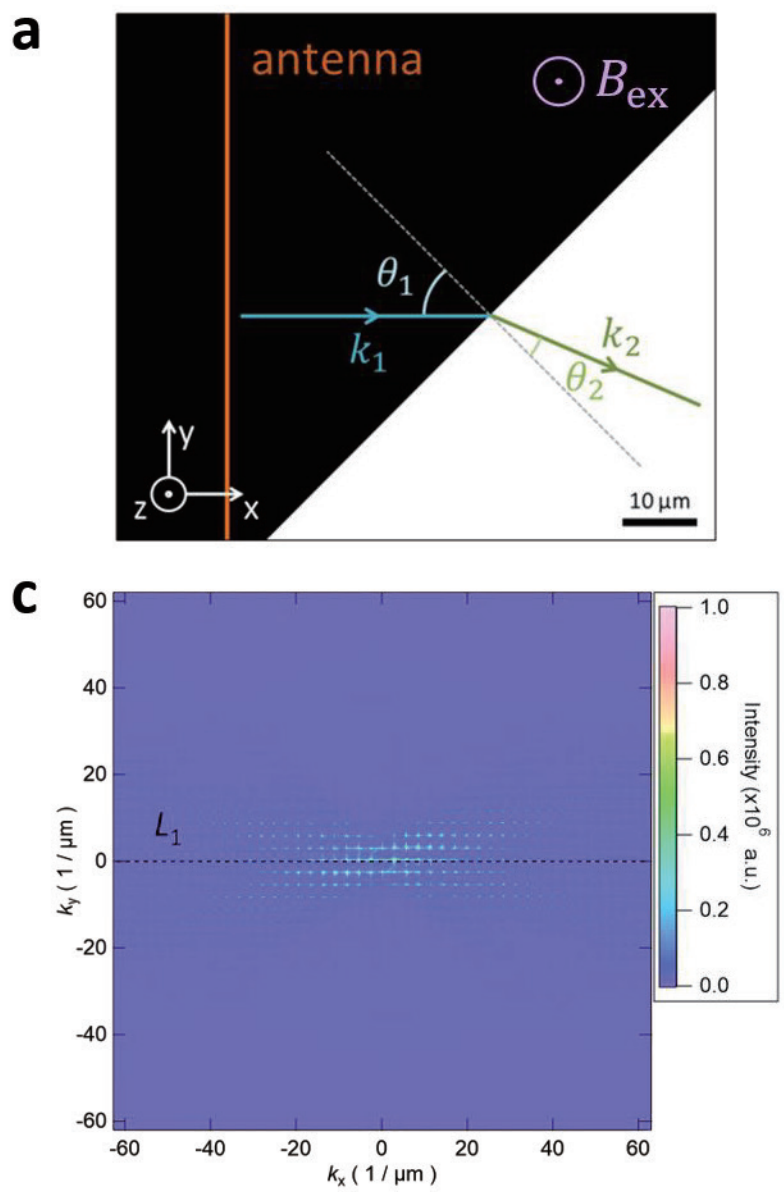

e

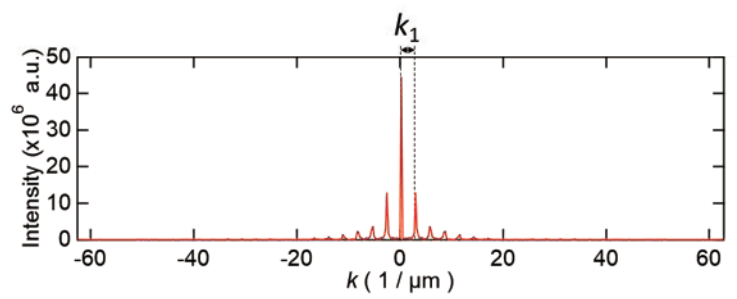

interaction and exchange interaction are taken into account. In the small- $k$ limit (i.e. for magnetostatic SWs), where $k^{2}$ is comparatively smaller than $k d$, the influence of exchange interaction can be ignored. In that case, the SW dispersion relation for out-of-plane magnetized films, named as magnetostatic forward volume wave (MSFVW), is expressed as

$$
\omega^{2}=\omega_{H}\left[\omega_{H}+\omega_{M}\left(1-\frac{1-e^{-k d}}{k d}\right)\right]
$$
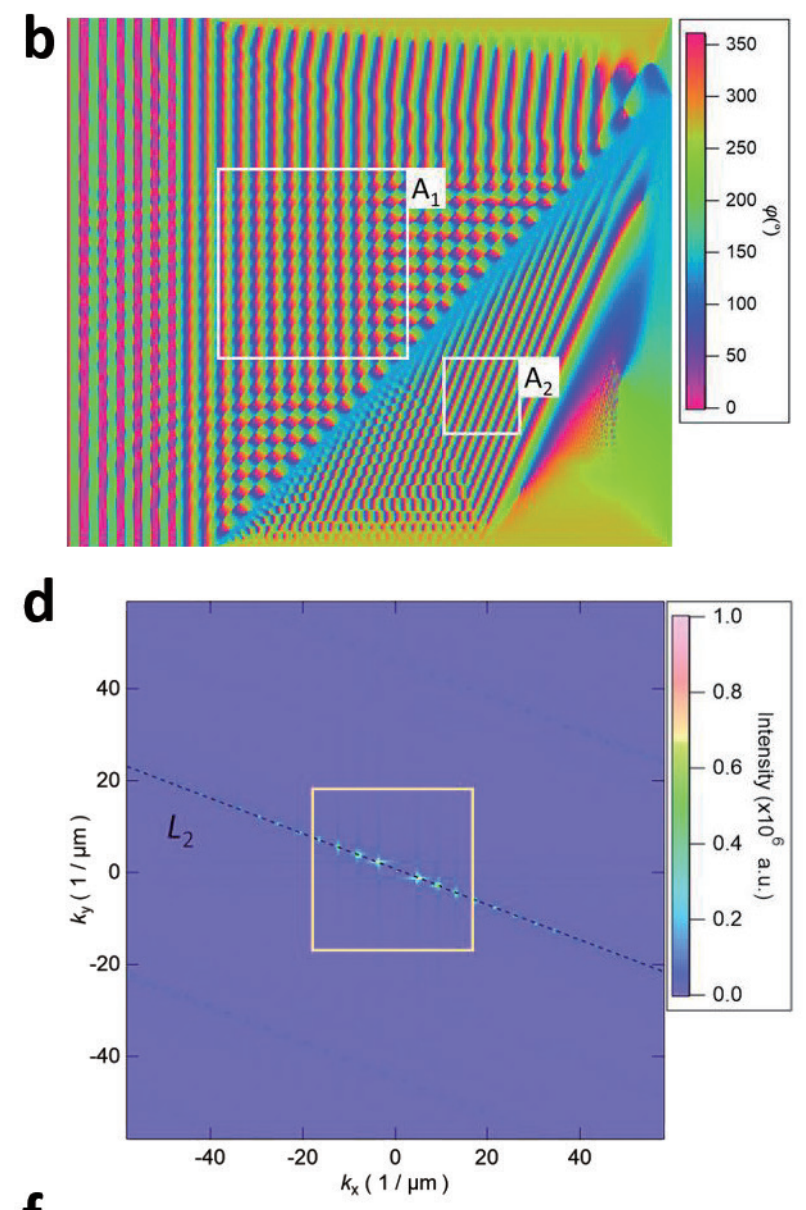

f

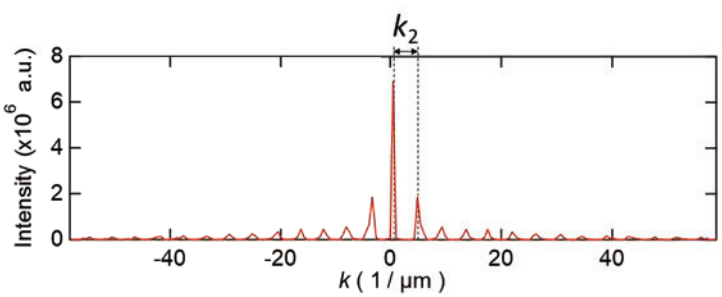

Fig.1 Example of process of simulation and analysis. The shown results are obtained from sample B when we set $f=1 \mathrm{GHz}$ and $\theta_{1}=45^{\circ}$. (a) Typical simulation setup. The black and white areas are respectively the thicker and thinner regions. The thickness step (boundary between two regions) is tilted with the angle $\theta_{1}$. The rf magnetic field is applied at the antenna (orange line), which has one-cell width. $k$ and $\theta$ of incident and refracted SWs are described by the blue and green arrows, respectively. (b) Simulation result of the SW propagation. The phase of magnetization precession, $\varphi$, is displayed. The areas $\mathrm{A}_{1}$ and $\mathrm{A}_{2}$ surrounded by white frames are utilized for analysis. (c,d) Results of 2D-FFT from (c) $A_{1}$ and (d) $A_{2}$. The black dashed line $\mathrm{L}_{1}$ in (c) is $k_{y}=0$ and $\mathrm{L}_{2}$ in (d) is the result of linear fitting of intensity in the yellow frame. (e,f) Intensity on (e) $\mathrm{L}_{1}$ and (f) $\mathrm{L}_{2}$. 

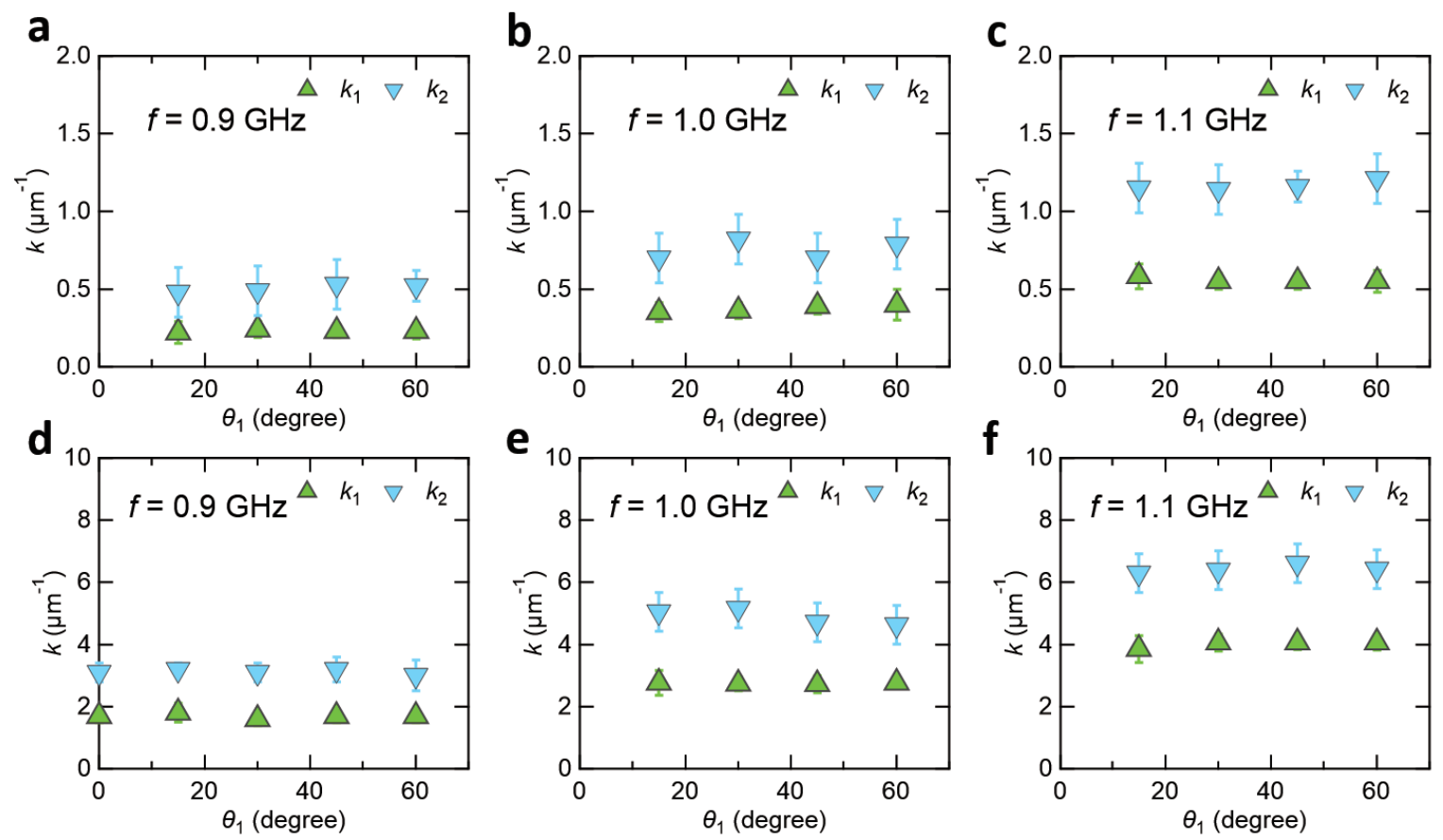

Fig.2 Incident angle dependent $k_{1}$ and $k_{2}$ in sample A (a,b,c) and sample B (d,e,f). The SWs are excited at three different resonant frequencies; $0.9 \mathrm{GHz}(\mathrm{a}, \mathrm{d}), 1.0 \mathrm{GHz}(\mathrm{b}, \mathrm{d})$, and $1.1 \mathrm{GHz}(\mathrm{c}, \mathrm{f})$. The green markers show $k_{1}$ and the blue markers show $k_{2}$.

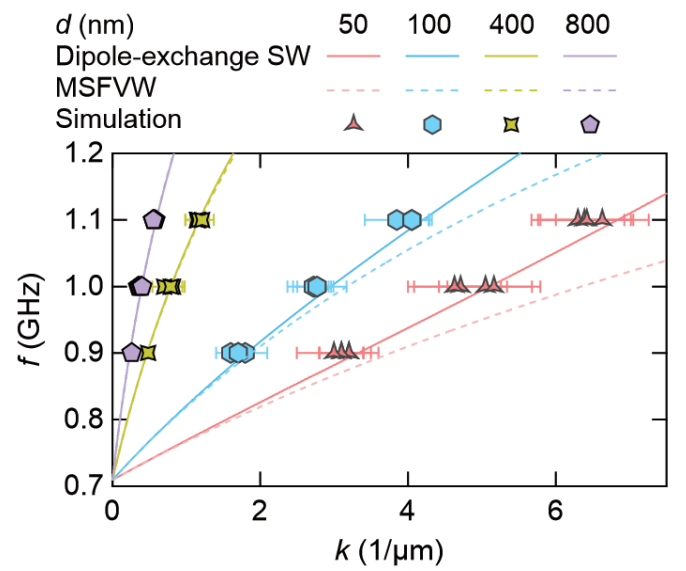

Fig.3 The resonant frequency as a function of wavenumber. Dispersion relation of DEFVW and MSFVW are respectively described by the solid and broken lines and the simulation results are plotted by the markers. Purple and green markers represent the SWs in the film of $d=800 \mathrm{~nm}$ and $400 \mathrm{~nm}$, referring to the data of $k_{1}$ and $k_{2}$ in sample $\mathrm{A}$, respectively. The blue and red markers represent the SWs in the film of $d=$ $100 \mathrm{~nm}$ and $50 \mathrm{~nm}$, referring to the data of $k_{1}$ and $k_{2}$ in sample B, respectively.

Fig. 3 shows the resonant frequency as a function of wavenumber. Symbols show the results by the micromagnetic simulation, and the calculation results by Eq. (1) and Eq. (2) are shown by broken lines and solid lines, respectively. While the simulation results in the regions of 400 and $800 \mathrm{~nm}$ thicknesses (sample A) are well reproduced by Eq. (2), those in the regions of 50 and $100 \mathrm{~nm}$ thicknesses (sample B) show deviation from Eq. (2) and can be explained by Eq. (1), indicating that not only the dipole interaction but also the exchange interaction should be taken into account to describe the refraction of spin waves with high wavenumbers.

Figs. 4(a) and 4(b) show the relation between the incident angle $\theta_{1}$ and the refraction angle $\theta_{2}$ observed in the samples $\mathrm{A}$ and $\mathrm{B}$, respectively. The refraction angle is independent of the resonant frequency in the sample A. This is due to the specific nature of MSFVW. According to Eq. (2) for MSFVW, the wavenumber varies by keeping $k d$ constant, resulting in the special conditon of $k_{1} / k_{2}=d_{2} / d_{1}$. Therefore, Snell's law, $\sin \theta_{1} / \sin \theta_{2}=$ $k_{2} / k_{1}$, is given solely by the thickness ratio of two regions. On the other hand, in the case of the dipole-exchange forward volume wave (DEFVW) described by Eq. (1), the ratio $k_{2} / k_{1}$ depends not only on the thickness ratio but also on the frequency. Note that similar effect is also well known in optics as the chromatic aberration due to the dependence of refractive index on wavelength of light. The lines in Figs. 4(a) and 4(b) show the calculation results of Snell's law based on Eq. (1) and on Eq. (2), respectively, and the simulation results well reproduce the calculation results, confirming that the refraction of SWs follows the Snell's law in optics with the chromatic aberration effect. 


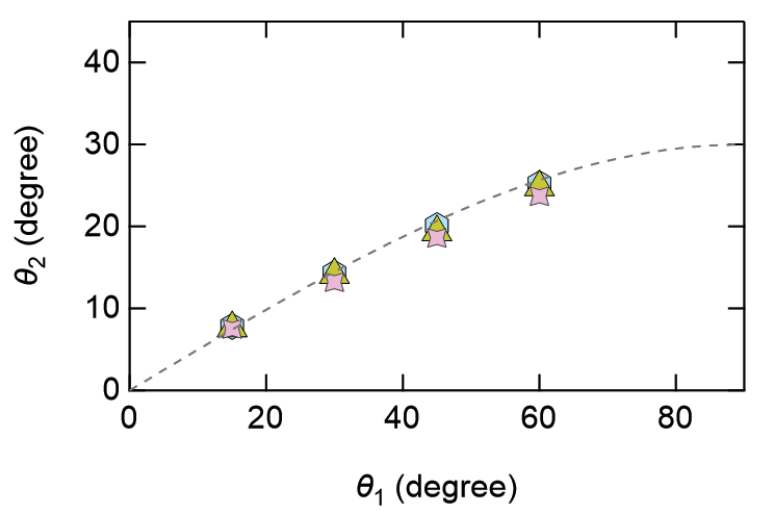

Calculation

$0.9,1.0,1.1 \mathrm{GHz}$

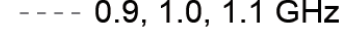

b

b Simulation

口. $0.9 \mathrm{GHz}$

$\triangle 1.0 \mathrm{GHz}$

- $1.1 \mathrm{GHz}$

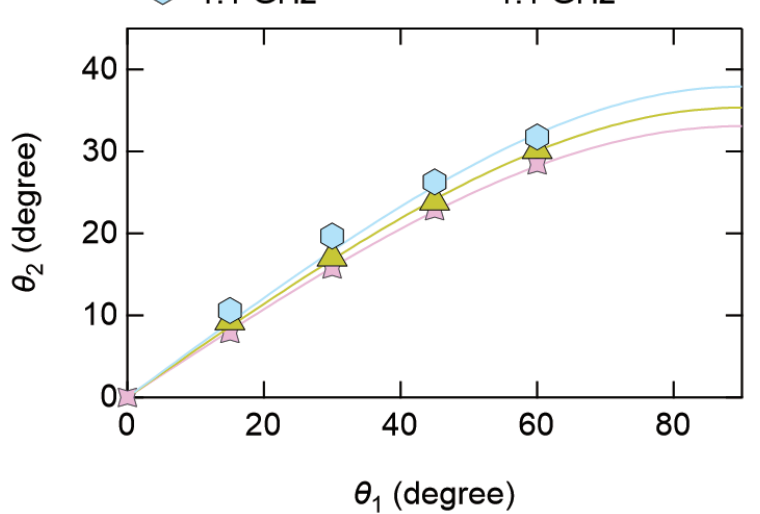

Fig.4 Refraction angle versus incident angle of the SWs propagating (a) in sample A and (b) in sample B. In both figures, the pink, green, and blue markers correspond to the simulation results of $f=0.9,1.0$, and $1.1 \mathrm{GHz}$, respectively. (a) Snell's law of MSFVW is expressed by the black dashed line. (b) The frequency dependent Snell's law of $0.9,1.0$, and $1.1 \mathrm{GHz}$ are shown by the pink, green, and blue solid lines, respectively.

In summary, we performed the micromagnetic simulations to investigate the refraction of SWs across the thickness step in magnetic thin films with out-ofplane magnetization. It was confirmed that the SWs propagate isotropically and the Snell's law in optics can be applied in the present system, leading to the simple design of magnonic devices. It was also found that while the refraction angle is independent of the resonant frequency in MSFVW it depends on the resonant frequency in the case of DEFVW, suggesting that the chromatic aberration effect should be taken into account in designing magnonic devices using spin waves with high wavenumbers.

Acknowledgements We gratefully acknowledge funding of JSPS KAKENHI Grant Numbers 15H05702, 18J22219, Collaborative Research Program of the Institute for Chemical Research, Kyoto University, and the Cooperative Research Project Program of the Research Institute of Electrical Communication, Tohoku University.

\section{References}

1) R. L. Stamps, S. Breitkreutz, J. Åkerman, A. V. Chumak, Y. Otani, G. E. W. Bauer, J. -U. Thiele, M. Bowen, S. A. Majetich, and M. Kläui: Journal of Physics D: Applied Physics, 47(33), 333001 (2014).

2) J. M. Owens, J. H. Collins, and R. L. Carter, Circuits; Systems and signal processing, 4(1-2), 317-334 (1985).

3) J. D. Adam: Proceedings of the IEEE, 76(2), 159-170 (1988).

4) A. V. Chumak, V. I. Vasyuchka, A. A. Serga, and B. Hillebrands: Nat. Phys., 11, 453 (2015).

5) P. Gruszecki, Yu. S. Dadoenkova, N. N. Dadoenkova, I. L. Lyubchanskii, J. Romero-Vivas, K. Y. Guslienko, and M. Krawczyk: Phys. Rev. B, 92, 054427 (2015).

6) P. Gruszecki, J. Romero-Vivas, Yu. S. Dadoenkova, N. N. Dadoenkova, I. L. Lyubchanskii, and M. Krawczyk: Appl. Phys. Lett., 105, 242406 (2014).
7) K. Yasumoto and Y. Ōishi: J. Appl. Phys., 54, 2170 (1983).

8) R. Gieniusz, V. D. Bessonov, U. Guzowska, A. I. Stognii, and A. Maziewski: Appl. Phys. Lett., 104, 082412 (2014).

9) Y. I. Gorobets and S. A. Reshetnyak, Technical Physics, 43(2), 188-191 (1998).

10) B. A. Kalinikos and A. N. Slavin: J. Phys. C: Solid State Phys., 19, 7013 (1986).

11) J. -N. Toedt, M. Mundkowski, D. Heitmann, S. Mendach, and W. Hansen: Sci. Rep., 6, 33169 (2016).

12) H. Hata, T. Moriyama, K. Tanabe, K. Kobayashi, R. Matsumoto, S. Murakami, J. -I. Ohe, D. Chiba, T. Ono: J. Magn. Soc. Jpn., 39, 151 (2015).

13) J. Stigloher, M. Decker, H. S. Körner, K. Tanabe, T. Moriyama, T. Taniguchi, H. Hata, M. Madami, G. Gubbiotti, K. Kobayashi, T. Ono, and C. H. Back: Phys. Rev. Lett., 117, 037204 (2016).

14) D. -E. Jeong, D. -S. Han, and S. -K. Kim: Spin, 1, 27-31 (2011).

15) G. Csaba, A. Papp, and W. Porod: J. Appl. Phys., 115, 17C741 (2014).

16) J. Stigloher, T. Taniguchi, M. Madami, M. Decker, H. S. Körner, T. Moriyama, G. Gubbiotti, T. Ono, and C. H. Back: Appl. Phys. Express, 11, 053002 (2018).

17) K. Tanabe, R. Matsumoto, J. -I. Ohe, S. Murakami, T. Moriyama, D. Chiba, K. Kobayashi, and T. Ono: Appl. Phys. Express, 7, 053001 (2014).

18) S. -K. Kim, S. Choi, K. -S. Lee, D. -S. Han, D. -E. Jung, and Y. -S. Choi: Appl. Phys. Lett., 92, 212501 (2008).

19) A. Vansteenkiste, J. Leliaert, M. Dvornik, M. Helsen, F. Garcia-Sanchez, and B. Van Waeyenberge: AIP Advances, 4, 107133 (2014).

20) A. S. KindyakV., A. KolosovL., and N. Makutina: j. Mater. Sci.: Mater. Electron., 6, 25-27 (1995).

21) A. V. Chumak, A. A. Serga, and B. Hillebrands: Nat. Commun., 5, 4700 (2014).

22) M. H. Seavey, Jr., and P. E. Tannenwald: Phys. Rev. Lett., 1, 168 (1958).

Received Aug. 27, 2020; Revised Sep. 18, 2020; Accepted Sep. 23, 2020 